\title{
Effects of Music Intervention on the Physical and Mental Status of Patients with Breast Cancer: A Systematic Review and Meta-Analysis
}

\author{
Xia Wang $^{\text {a }}$ Yin Zhang $^{a}$ Yao Fan ${ }^{b, c, d}$ Xiang-Sheng Tan ${ }^{\mathrm{e}} \quad$ Xun Lei ${ }^{\mathrm{b}, \mathrm{c}, \mathrm{d}}$ \\ a School of Pediatrics, Chongqing Medical University, Chongqing, China; \\ bSchool of Public Health and Management, Chongqing Medical University, Chongqing, China; \\ ${ }^{\mathrm{c}}$ Research Center for Medicine and Social Development, Chongqing Medical University, Chongqing, China; \\ ${ }^{d}$ Collaborative Innovation Center of Social Risks Governance in Health, Chongqing Medical University, Chongqing, China; \\ ${ }^{e}$ Fifth Clinical College, Chongqing Medical University, Chongqing, China
}

\section{Keywords}

Music intervention · Breast neoplasms · Meta-analysis

\section{Summary}

Background: The aim of this review was to evaluate the effects of music intervention on the physical and mental status of patients with breast cancer. Methods: 9 databases were searched; 30 randomized controlled trials that compared the effects of music plus standard care and standard care alone in patients with breast cancer were included. Methodological quality was assessed using the Jadad scale. Results: The results of the metaanalysis suggested that music intervention was significantly effective in lowering systolic blood pressure (standardized mean difference (SMD) -0.63, 95\% confidence interval (Cl) -0.85 to $-0.42 ; \mathrm{p}<0.00001$ ), diastolic blood pressure (SMD $-0.64,95 \% \mathrm{Cl}-1.06$ to $-0.22 ; \mathrm{p}=$ 0.003 ), and heart rate (SMD $-0.45,95 \% \mathrm{Cl}-0.66$ to -0.24 ; $\mathrm{p}<0.0001$ ), and in relieving anxiety (Hamilton Scale: mean difference (MD) $-7.04,95 \% \mathrm{Cl}-9.31$ to $-4.78 ; \mathrm{p}$ < 0.00001; Self-Rating Anxiety Scale: MD -7.40, 95\% Cl -10.28 to $-4.52 ; p<0.00001$; State Anxiety Inventory: MD $-12.40,95 \% \mathrm{Cl}-21.86$ to $-2.94 ; \mathrm{p}=0.01$ ), and depression (MD -7.39, 95\% Cl -8.35 to -6.43 ; $\mathrm{p}<0.00001$ ). Conclusion: This review provides clues that music intervention might partially improve the physiological and psychological health of patients with breast cancer.

(C) 2018 S. Karger GmbH, Freiburg

\section{Introduction}

Breast cancer is the most frequently diagnosed cancer and the leading cause of cancer death among females worldwide, accounting for $25 \%$ of all cancer cases and $15 \%$ of all cancer deaths among women, with an estimated 1.7 million cases and 521,900 deaths in 2012 [1]. Breast cancer has caused a heavy social burden, and is becoming a serious health problem in both developing and developed countries [2]. Patients with breast cancer may undergo severe physical and psychological trauma, including impaired body image, breast loss, fatigue, insomnia, depression [3-9], pain [1014], and other negative emotions. Those traumata could have negative effects on long-term quality of life in breast cancer survivors [15].

Breast cancer treatment usually begins with surgery followed by various combinations of adjuvant therapies involving radio- and chemotherapy as well as hormone therapy [16]. Surgery and pharmaceutical therapy can be effective but may have side effects and secondary influences that lead to negative psychological effects. Patients with breast cancer are more likely to have symptoms of depression and anxiety $[9,17]$. Depression, in turn, can lead to fatigue, sleeping difficulties, digestive problems, pathological worry, prolonged arousal, lowered immune function, feelings of helplessness, and pessimism [18]. Serious anxiety might induce patients to experience high blood pressure and increased heart rate [13, 19]. In addition, women who undergo surgery for breast cancer often experience postoperative pain that can cause considerable physical disability and psychological disturbances [20].

The most common ways to manage the mental status of patients with breast cancer are cognitive-behavioral therapy and art

\section{KARGER}

(๑) 2018 S. Karger GmbH, Freiburg 
therapy, which both include music, guided imagery, mindfulness meditation, and relaxing exercise training. Among those approaches, music intervention is highly recommended. Music interventions are categorized as music medicine and music therapy. Music medicine is the passive listening to prerecorded music offered by medical personnel with no systematic therapeutic process. Music therapy on the other hand is implemented by trained music therapists with all kinds of experience in the field of music; it is a systematic therapeutic process including assessment, treatment, and evaluation [21]. Both music medicine and music therapy were assessed in previous studies. Music has a great impact on mood, and sometimes it can alter the way a patient copes with and feels about a disease [22]. The effectiveness of music intervention for patients with breast cancer has been documented in both descriptive and experimental studies, as music intervention has been broadly applied. Music can ameliorate negative emotions such as anxiety $[3,5,19,23]$, depression [3-7], and pain [10, 12-14] before, during, and after the therapeutic process. During the administration of chemotherapy, integrative intervention with music has been rated as helpful for women with breast cancer to reduce nausea and vomiting [24-31]. In addition, musical rhythms can structure behavior by simultaneously and directly influencing emotions and altering physiological functioning, and slow music can decrease blood pressure, heart rate, and respiratory rate $[19,32-34]$.

While there have been large amounts of studies proving that music intervention is effective for improving the physical and mental status of patients with breast cancer, there has not yet been a systematic and comprehensive review or a meta-analysis to evaluate the effects of music intervention on breast cancer patients. In 2014, Boehm et al. [35] carried out a systematic review which included the effects of art therapy on breast cancer patients. Music was considered only as a component of art therapies, but not as an individual intervention. In 2011, Bradt et al. [36] published a meta-analysis assessing the effectiveness of music intervention in cancer patients. The researchers concluded that music interventions may have beneficial effects on anxiety, pain, mood, and quality of life in cancer patients, and a small effect on heart rate, respiratory rate, and blood pressure. While breast cancer was included in their review, no separate analysis on breast cancer patients was carried out. Moreover, all related original studies suffer from small sample size, making outcomes difficult to gauge accurately $[3-14,17,19,23,24,37-40]$. In addition, patient groups in these original studies were often heterogeneous, with differences in factors such as study design, method of intervention, and intensity of treatment leading to varying results.

The objective of this systematic review and meta-analysis is to summarize evidence and evaluate the effects of music intervention on the physical and mental status of patients with breast cancer. The findings from this study are expected to make an important contribution to treatment guidelines for breast cancer patients.

\section{Methods}

Search Strategy

An electronic search of 9 databases (PubMed, CINAHL, Cochrane, Embase, Web of Science, Sinomed, VIP, CNKI, and Wanfang Data) was conducted from their start date to September 2017. For English databases, the key words 'music', 'breast cancer', 'breast neoplasms', 'breast carcinoma', 'breast tumor', and 'mammary cancer' were combined. For Chinese databases, corresponding Chinese terms for breast cancer and music were used.

Criteria for Inclusion and Exclusion

For eligible studies, inclusion criteria were as follows: i) included patients with breast cancer; ii) included music as the intervention; iii) compared music intervention plus routine care versus routine care alone, not including self-control; iv) assessed complete outcomes (including pre-test and post-test outcomes); v) designed as a randomized controlled trial (RCT); and vi) published in English or Chinese. Exclusion criteria were as follows: i) included breast cancer patients with other diseases; ii) included music and other types of intervention; iii) designed as a study other than an RCT (e.g., review, survey, case study, meeting report, controlled clinical trial); iv) the full-text article was not accessible; and v) was a duplicate (only the article with the most detailed information was included).

\section{Data Extraction}

Data were extracted independently by the 2 first authors using a self-designed data extraction sheet to determine the study characteristics (supplementary table 1, available at http://www.karger.com/ProdukteDB/produkte. asp?doi=487073). A discussion was held upon any differences until agreement was reached between the authors on the data extracted before data analysis. Where possible, results for the meta-analysis were presented using mean difference (MD) and standardized mean difference (SMD). Otherwise, a descriptive analysis of the study was carried out.

\section{Assessment of Methodological Quality}

The 2 first authors (WX, ZY) independently assessed the quality of the included studies according to the criteria of the Jadad scale [41]. The assessment of risk of bias includes 4 items: i) randomization, ii) concealment of allocation, iii) double blinding, iv) withdrawals and dropouts. The results of the assessment are presented as appropriate ( 2 points), unclear ( 1 points), or inappropriate ( 0 point) for the first 3 items. For item 4 , if the study included a description of the patient withdrawals or there was no withdrawal or dropout, it got 1 point; otherwise it got 0 points. The maximum score is thus 7 points. It is widely recognized that a score of $0-3$ points indicates a low-quality study, whereas a score of 4-7 points indicates a high-quality study.

\section{Data Synthesis and Statistical Analysis}

In this review, outcomes were presented as continuous variables. MD and SMD were used for continuous variables. We calculated $95 \%$ confidence intervals $(95 \% \mathrm{CI})$ for each effect size estimate, and a random-effects model or fixedeffects model was used. A p value $<0.05$ was considered to be statistically significant. Heterogeneity among studies was tested using the $\mathrm{I}^{2}$ index which describes the percentage of total variation across studies that is due to heterogeneity rather than to chance. A percentage of around $25 \%\left(\mathrm{I}^{2}=25\right), 50 \%\left(\mathrm{I}^{2}=50\right)$, and $75 \%\left(\mathrm{I}^{2}=75\right)$ indicates low, medium, and high heterogeneity, respectively. A subgroup analysis was conducted to assess possible sources of heterogeneity. Significant heterogeneity between subgroups indicates that the subgroup factor may explain part of the total heterogeneity. The following treatment comparison was made: music interventions plus standard care (experimental group) versus standard care alone (control group). All statistical analyses were performed using Review Manager 5.0 [42]. 
Fig. 1. Comparison music versus control outcome systolic blood pressure.

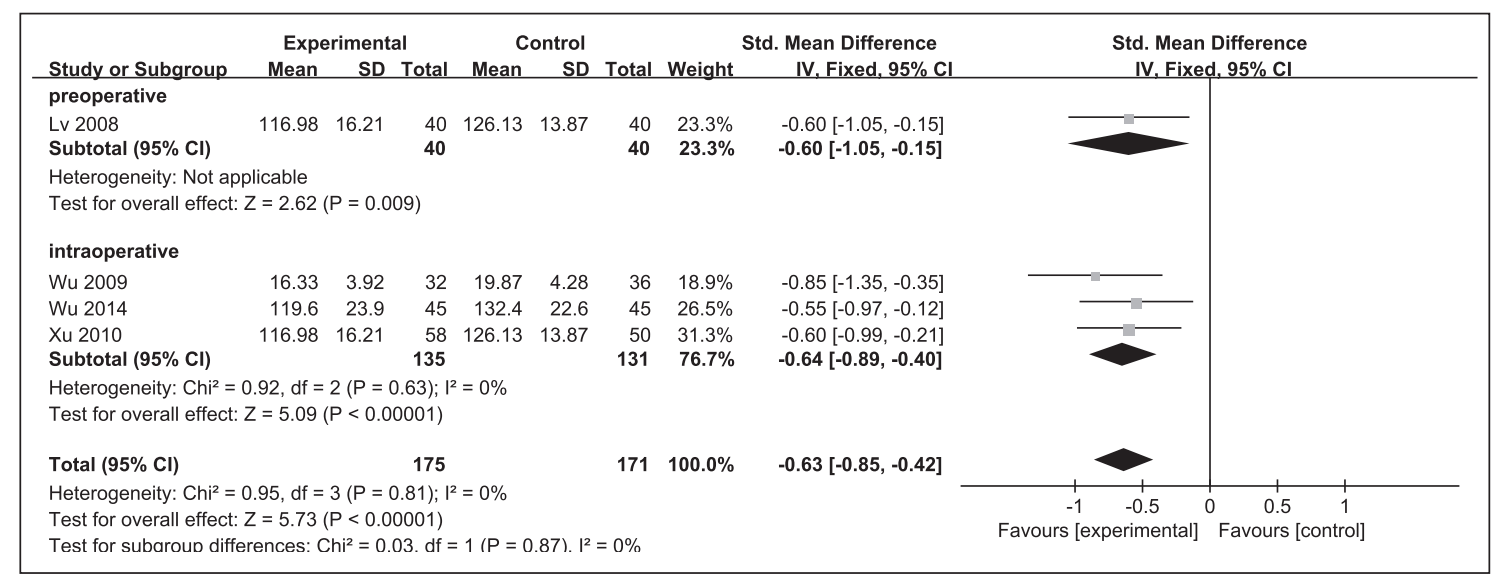

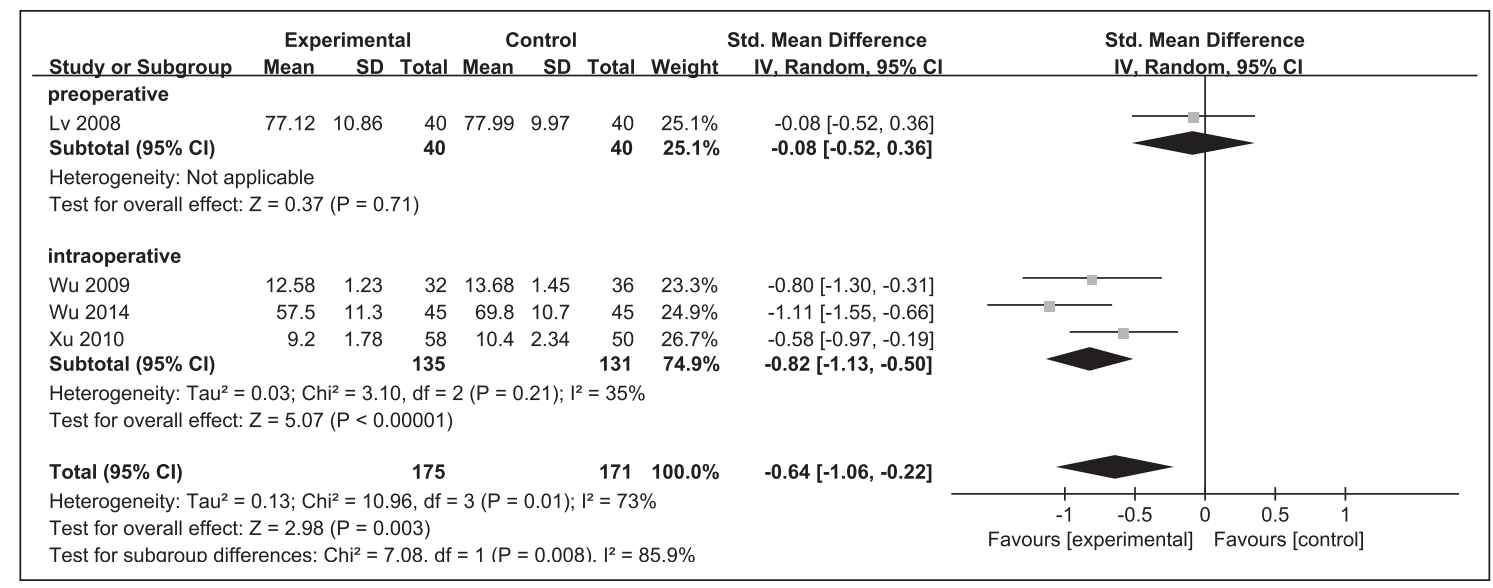

Fig. 2. Comparison music versus control outcome diastolic blood pressure.

\section{Results}

\section{Study Selection}

Initial queries yielded a total of 860 studies, but only 637 studies were left after duplicates were removed. Following this, titles and abstracts were reviewed for the 637 studies, of which 560 were deemed ineligible for various reasons. The full text of the remaining 77 studies was read, and then 47 of those studies were eliminated because of failure to meet the inclusion criteria. In total, 30 studies met all eligibility criteria and were included in the present study.

\section{Characteristics of Included Studies}

The literature search revealed 30 RCTs with a total of $n=2,559$ breast cancer patients (1,292 in the experimental group vs. 1,267 in the control group). All studies were carried out between 2002 and 2017. 6 studies were presented in English [9, 12-14, 17, 40]; the remaining studies were presented in Chinese. The age of the patients ranged from 18 to 75 years. Sample sizes ranged from 30 to 310 . Treatments varied across studies: 22 studies reported that patients received chemotherapy [3-9, 12, 14, 17, 24-31, 37-40], which was sometimes in combination with other treatments; 17 studies reported that patients received mastectomy $[3,6,7,9,10,12,13,17$,
$23,27,30-34,37,38]$; other studies mentioned only routine nursing care. A complete list of the characteristics of the included studies is shown in supplementary table 1 (available at $h t t p: / / w w w$. karger.com/ProdukteDB/produkte.asp?doi=487073).

\section{Methodological Quality}

The methodological quality of the included studies was assessed according to the Jadad scale. 8 studies were rated at 4 points $[5,7$, $10,13,34,37,38,40]$ and 1 study at 5 points [14]; these were thus considered high-quality studies. 17 studies received 3 points, and the remaining 4 studies received only 1 point; these were thus considered low-quality studies.

\section{Meta-Analysis}

\section{Physiological Outcomes}

\section{Systolic Blood Pressure}

Four trials, which included a total of 346 patients, were used to produce a fixed-effects model for systolic blood pressure [19, 3234]. The overall SMD was -0.63 ( $95 \%$ CI -0.85 to -0.42 ; p $<0.00001$ ) (fig. 1). There was no study heterogeneity in this analysis $\left(\mathrm{I}^{2}=0 \% ; \mathrm{p}=0.81\right)$. 
Fig. 3. Comparison music versus control outcome heart rate.

Fig. 4. Comparison music versus control outcome Hamilton Anxiety-Rating Scale.

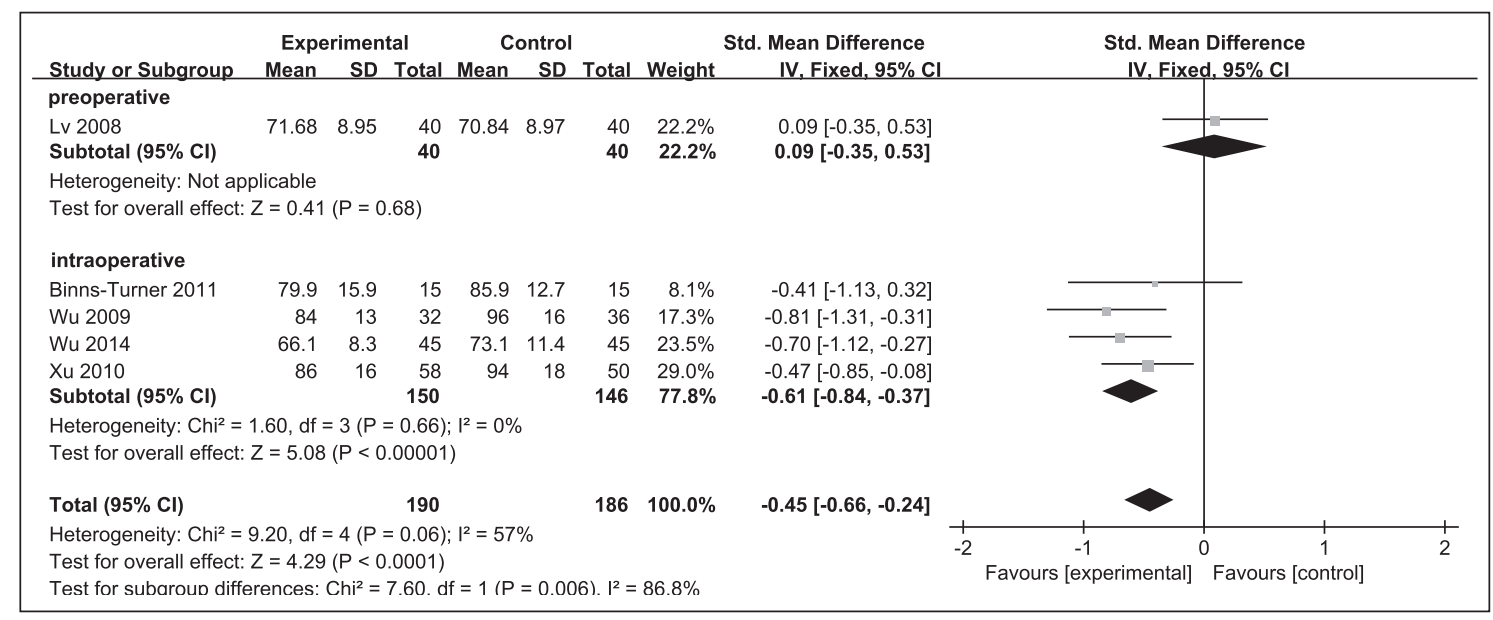

\begin{tabular}{|c|c|c|c|c|c|c|c|c|c|c|c|}
\hline \multirow[b]{2}{*}{ Study or Subgroup } & \multicolumn{3}{|c|}{ Experimental } & \multicolumn{2}{|c|}{ Control } & \multirow[b]{2}{*}{ Total } & & \multirow{2}{*}{$\begin{array}{l}\text { Mean Difference } \\
\text { IV. Random, } 95 \% \mathrm{Cl}\end{array}$} & \multirow{2}{*}{\multicolumn{3}{|c|}{$\begin{array}{l}\text { Mean Difference } \\
\text { IV, Random, } 95 \% \mathrm{CI}\end{array}$}} \\
\hline & Mean & SD & Total & Mean & SD & & Weight & & & & \\
\hline $\operatorname{Jin} 2008$ & 15.57 & 3.63 & 50 & 21.44 & 2.51 & 50 & $49.2 \%$ & $-5.87[-7.09,-4.65]$ & - & & \\
\hline Ma 2013 & 14.97 & 3.56 & 50 & 23.15 & 1.62 & 50 & $50.8 \%$ & $-8.18[-9.26,-7.10]$ & & & \\
\hline Total $(95 \% \mathrm{Cl})$ & & & 100 & & & 100 & $100.0 \%$ & $-7.04[-9.31,-4.78]$ & & & \\
\hline \multicolumn{9}{|c|}{$\begin{array}{l}\text { Heterogeneity: } \mathrm{Tau}^{2}=2.32 ; \mathrm{Chi}^{2}=7.67, \mathrm{df}=1 \\
\text { Test for overall effect: } \mathrm{Z}=6.10(\mathrm{P}<0.00001)\end{array}$} & -10 & 5 & 10 \\
\hline
\end{tabular}

\begin{tabular}{|c|c|c|c|c|c|c|c|c|c|c|}
\hline \multirow[b]{2}{*}{ Study or Subgroup } & \multicolumn{3}{|c|}{ Experimental } & \multicolumn{2}{|c|}{ Control } & \multirow[b]{2}{*}{ Total } & \multirow[b]{2}{*}{ Weight } & \multirow{2}{*}{$\begin{array}{l}\text { Mean Difference } \\
\text { IV, Random, } 95 \% \mathrm{Cl}\end{array}$} & \multirow{2}{*}{\multicolumn{2}{|c|}{$\begin{array}{l}\text { Mean Difference } \\
\text { IV, Random, } 95 \% \mathrm{CI}\end{array}$}} \\
\hline & Mean & SD & Total & Mean & SD & & & & & \\
\hline \multicolumn{11}{|l|}{ low quality } \\
\hline $\begin{array}{l}\text { Fu } 2009 \\
\text { Subtotal }(95 \% \mathrm{Cl})\end{array}$ & 24.9 & 5.52 & $\begin{array}{l}40 \\
40\end{array}$ & 26.18 & 5.48 & $\begin{array}{l}38 \\
38\end{array}$ & $\begin{array}{l}19.7 \% \\
19.7 \%\end{array}$ & $\begin{array}{l}-1.28[-3.72,1.16] \\
-1.28[-3.72,1.16]\end{array}$ & & \\
\hline \multicolumn{11}{|c|}{ Heterogeneity: Not applicable } \\
\hline \multicolumn{11}{|l|}{ high quality } \\
\hline Gu 2015 & 46.91 & 6.46 & 40 & 58.76 & 4.78 & 40 & $19.6 \%$ & $-11.85[-14.34,-9.36]$ & & \\
\hline Sun 2013 & 43.48 & 3.39 & 30 & 51.56 & 1.86 & 30 & $21.8 \%$ & $-8.08[-9.46,-6.70]$ & - - & \\
\hline Wu 2014 & 35.4 & 2.7 & 45 & 44.3 & 4.3 & 45 & $21.6 \%$ & $-8.90[-10.38,-7.42]$ & - - & \\
\hline Zhou 2009 & 37.9 & 5.51 & 30 & 44.5 & 7.69 & 30 & $17.4 \%$ & $-6.60[-9.99,-3.21]$ & & \\
\hline Subtotal $(95 \% \mathrm{Cl})$ & & & 145 & & & 145 & $80.3 \%$ & $-8.92[-10.59,-7.25]$ & & \\
\hline \multicolumn{11}{|c|}{$\begin{array}{l}\text { Heterogeneity: } \mathrm{Tau}^{2}=1.76 ; \mathrm{Chi}^{2}=8.44, \mathrm{df}=3(P=0.04) ; I^{2}=64 \% \\
\text { Test for overall effect: } Z=10.48(P<0.00001)\end{array}$} \\
\hline Total $(95 \% \mathrm{Cl})$ & & & 185 & & & 183 & $100.0 \%$ & $-7.40[-10.28,-4.52]$ & & \\
\hline $\begin{array}{l}\text { Heterogeneity: } \mathrm{Tau}^{2}= \\
\text { Test for overall effect: } \\
\text { Test for subaroun diffe }\end{array}$ & $\begin{array}{l}9.40 ; \mathrm{Ch} \\
=5.04 \\
\text { rences: }\end{array}$ & $\begin{array}{l}\mathrm{i}^{2}=40 . \\
(P<0 . \\
\mathrm{Chi}^{2}=2\end{array}$ & $\begin{array}{l}27, \mathrm{df}= \\
00001) \\
25.64\end{array}$ & $\begin{array}{l}=4(P< \\
d f=1(F\end{array}$ & $\begin{array}{l}<0.000 \\
P<0.0\end{array}$ & $001) ; 1^{2}=$ & $\begin{array}{l}=90 \% \\
I^{2}=96.1 \%\end{array}$ & & $\begin{array}{ccc}-10 & -5 & 0 \\
\text { Favours [experimental] }\end{array}$ & $\begin{array}{ll}5 & 10 \\
\text { Favours [control] }\end{array}$ \\
\hline
\end{tabular}

Fig. 5. Comparison music versus control outcome Self-Rating Anxiety Scale.

\section{Psychological Outcomes}

Four trials, which included a total of 346 patients, were used to produce a random-effects model for diastolic blood pressure [19, $32-34]$. The overall SMD was -0.64 (95\% CI -1.06 to $-0.22 ; \mathrm{p}=$ 0.003 ) (fig. 2). There was study heterogeneity in this analysis $\left(\mathrm{I}^{2}=\right.$ $73 \% ; \mathrm{p}=0.01)$.

One study measured mean arterial pressure; as raw data could not be merged into the above data, this was not included in the meta-analysis [14].

\section{Heart Rate}

Five trials, which included a total of 376 patients, were used to produce a fixed-effects model for heart rate [13, 19, 32-34]. The overall SMD was -0.45 ( $95 \%$ CI -0.66 to -0.24 ; $\mathrm{p}<0.0001$ ) (fig. 3 ). There was study heterogeneity in this analysis $\left(\mathrm{I}^{2}=57 \%\right.$; $\left.\mathrm{p}=0.06\right)$. One study was excluded from this meta-analysis as its raw data was presented as a chart [14].

\section{Anxiety}

Two trials that relied on the Hamilton Anxiety-Rating Scale to measure anxiety and included a total of 200 patients were used to produce a random-effects model for anxiety [30, 31]. The overall MD was -7.04 (95\% CI -9.31 to $-4.78 ; \mathrm{p}<0.00001)$ (fig. 4$)$. There was study heterogeneity in this analysis $\left(\mathrm{I}^{2}=87 \% ; \mathrm{p}=0.006\right)$.

One trial was excluded from the meta-analysis because the raw data could not be merged with that from other trials [23]; thus only 5 trials were included in the meta-analysis that used the Self-Rating Anxiety Scale to analyze anxiety $[3,5,8,11,19]$. The 5 trials, which included a total of 368 patients, were used to produce a randomeffects model for anxiety. The overall MD was -7.40 (95\% CI -10.28 to $-4.52 ; \mathrm{p}<0.00001$ ) (fig. 5). There was study heterogeneity in this analysis $\left(\mathrm{I}^{2}=90 \% ; \mathrm{p}<0.00001\right)$. 
Fig. 6. Comparison music versus control outcome State Anxiety Inventory.

Fig. 7. Comparison music versus control outcome Self-Rating Depression Scale.

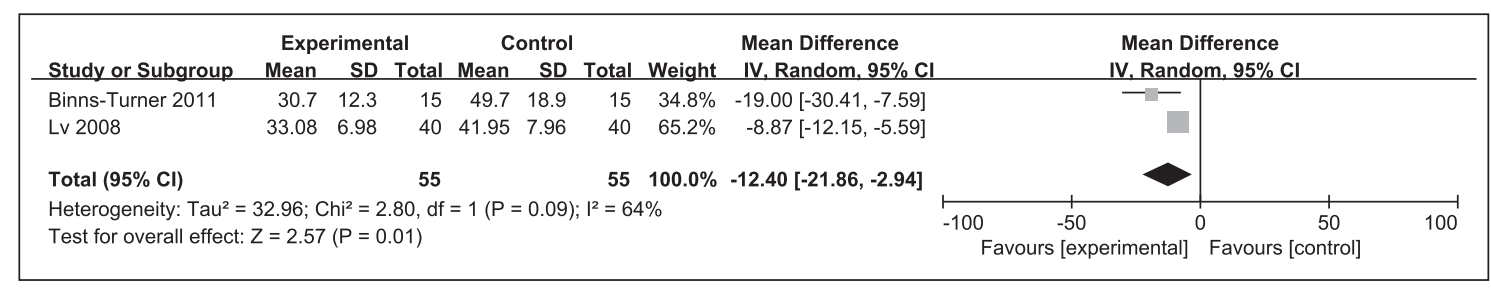

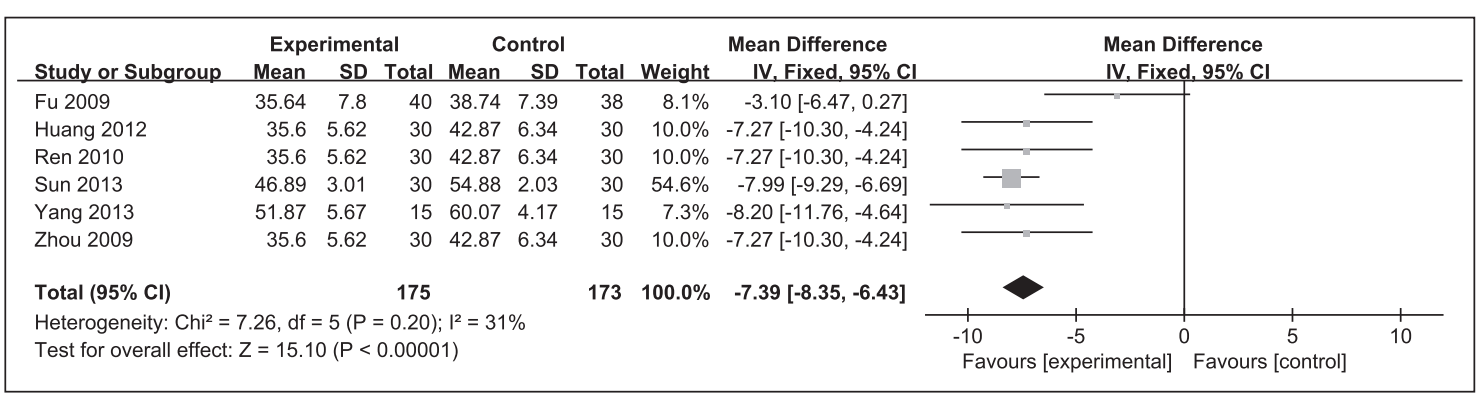

Although there were 5 studies that used the State Anxiety Inventory to assess anxiety, 3 studies were excluded from the metaanalysis: 2 studies tested anxiety in every chemotherapy duration $[17,37]$, and 1 study presented data in chart format [40]; thus they could not be merged with others. 2 trials, which included a total of 110 patients, were used to produce a random-effects model for State Anxiety Inventory [13, 34]. The overall MD was -12.40 (95\% $\mathrm{CI}-21.86$ to $-2.94 ; \mathrm{p}=0.01$ ) (fig. 6). There was study heterogeneity in this analysis $\left(\mathrm{I}^{2}=64 \% ; \mathrm{p}=0.09\right)$.

\section{Depression}

Seven trials reported the effects of music interventions on depression using the Self-Rating Depression Scale; however, one of these was excluded from the meta-analysis because the raw data could not be merged with that from the other trials [9]. The 6 remaining trials, which included a total of 348 patients, were used to produce a fixed-effects model for depression [3-8]. The overall MD was -7.39 (95\% CI -8.35 to $-6.43 ; \mathrm{p}<0.00001)$ (fig. 7). There was study heterogeneity in this analysis $\left(\mathrm{I}^{2}=31 \%\right.$; $\mathrm{p}=$ $0.20)$.

\section{Subgroup Analysis}

\section{Timing of Music Intervention}

For systolic blood pressure, the overall SMDs of studies with preoperative music intervention and those with intraoperative music intervention were $-0.60(95 \% \mathrm{CI}-1.05$ to -0.15$)$ and -0.64 (95\% CI -0.89 to $-0.40 ; \mathrm{p}<0.00001 ; \mathrm{I}^{2}=0 \%$ ), respectively (fig. 1 ).

For diastolic blood pressure, the overall SMDs of studies with preoperative music intervention and those with intraoperative music intervention were -0.08 (95\% CI -0.35 to 0.53$)$ and -0.82 (95\% CI -1.13 to $-0.50 ; \mathrm{p}<0.00001 ; \mathrm{I}^{2}=35 \%$ ), respectively (fig. 2).

For heart rate, the overall SMDs of studies with preoperative music intervention and those with intraoperative music intervention were 0.09 (95\% CI -0.35 to 0.53$)$ and -0.61 (95\% CI -0.84 to $-0.37 ; \mathrm{p}<0.00001 ; \mathrm{I}^{2}=0 \%$ ), respectively (fig. 3 ).
Differentiated Level of Methodological Quality

For anxiety examined by the Self-Rating Anxiety Scale, the overall MDs of low-quality studies and high-quality studies were $-1.28(95 \% \mathrm{CI}-3.72$ to $1.16 ; \mathrm{p}=0.30)$ and $-8.92(95 \% \mathrm{CI}-10.59$ to $-7.25 ; \mathrm{p}<0.00001 ; \mathrm{I}^{2}=64 \%$ ), respectively (fig. 5).

\section{Discussion}

\section{Effects of Music Intervention}

We carried out a systematic and comprehensive meta-analysis to examine the effects of music intervention on patients with breast cancer. Systolic blood pressure, diastolic blood pressure, heart rate, anxiety, and depression were significantly improved after music intervention as compared with the control group. This meta-analysis indicated that music intervention could partially improve the physiological and psychological health of patients with breast cancer.

Although 8 studies reported the effects of music intervention on nausea and vomiting, we could not carry out a meta-analysis of this research, as the raw data from these studies could not be merged due to different data presentation styles [24-31]. However, all 8 studies reported significantly less nausea and vomiting in the music intervention group as compared with the control group ( $p$ $<0.05$ ), which suggested that music can effectively improve gastroenteric function among patients with breast cancer. 5 additional studies reported the effects of music intervention on pain [10-14]. Data could not be merged because of the different scales used. Clinical findings from all 5 studies indicated that music intervention can effectively reduce pain in patients with breast cancer. However, additional research is needed to conduct a quantitative analysis to prove this result. Based on the above, we strongly suggest consistent scales or statistical methodology to be used in future research to allow data merging. Other outcomes such as duration of hospital stay [9], level of salivary cortisol [34], Quality of Life Questionnaire for Chinese Cancer patients receiving Chemobiotherapy [39], Karnofsky Performance Status [39], immunological 
function [38], the Rotterdam Symptom Checklist [37], and respiratory rate [32] were also reported. Although positive effects of music intervention were generally shown, more evidence is urgently needed to confirm these effects.

Although the mechanisms related to the effects of music intervention are not clear, it may have an impact on the function of the autonomic nervous system to regulate the excitability of the sympathetic and parasympathetic nervous system, resulting in a change in internal organ function [43]. Consequently, systolic blood pressure, diastolic blood pressure, heart rate, nausea, and vomiting may be improved by music intervention. Music affects the human reticular formation and limbic system, regulating emotional behavior and emotional experiences such as anxiety, depression, and pain [43]. Additionally, the positive effects of music on emotion are related to the release of neurotransmitters (e.g., serotonin and dopamine) [44].

Substantial heterogeneity was observed among these studies. The different timing of the music intervention and different levels of methodological quality are the main reasons for heterogeneity. In addition, the characteristics of the study participants - such as age, sex, culture, education, and interests - as well as their therapists' characteristics, the study design, and the intervention protocol may also have contributed to the heterogeneity in the metaanalysis. Those factors should be considered and consistent methodology should be adopted in future research to decrease heterogeneity across studies and produce effective results for evidence-based exploration.

\section{Application of Music}

Music intervention techniques include receptive music listening and music recreation and improvisation such as singing or playing instruments [45]. Receptive music listening was most frequently used in the included studies. This approach is easy to carry out given the limitations of time and performance space in the clinical setting. In addition, listening to music is relaxing and comfortable during chemotherapy. All patients were required to put on headsets to choose their preferred track and block out background noise during listening. However, the potential negative impact of the use of headphones during procedures should be considered as it may hamper the communication between the patient and the medical personnel. Some patients undergoing chemotherapy find headphones to be uncomfortable if they have lost their hair. We suggest the use of special speakers in future research which share music softly as an additional choice for music intervention.

Recreation and improvisation in music therapy have some therapeutic effects that differ from those of listening to music. The use of recreation and improvisation in an interactive way could help patients express emotional, physical, or psychological needs and difficulties, and find self-support and confidence to face their disease [45]. We strongly suggest that researchers consider recreation and improvisation in music intervention for breast cancer patients.
In the included studies, all music interventions were implemented by trained researchers. However, based on the limited information, we could not determine whether these were professional music therapists. Music therapists should be equipped with the knowledge of and practical experience in both musical and clinical fields, contributing to the successful application of music therapy techniques for patients. Bradt et al. [36] noted that music therapists are specially trained academically and clinically to select music interventions professionally to offer emotional and spiritual support, support the communication with loved ones, enhance the sense of control, and improve physical well-being in patients. Additionally, with the scientific knowledge of rigorously measuring process and outcome, music therapists have gained wider acceptance and greater significance in medical care. We suggest that music therapists should be more involved in clinical music intervention with better therapeutic schemes and effects evaluation.

The therapeutic relationship is also a crucial factor. A harmonious therapeutic relationship helps to maintain patient compliance, resulting in positive effects. Patient preference is pivotal in the therapeutic relationship, and the will of the patient typically decides the ultimate effects of the intervention. When listening to favorite pieces of music, positive reactions might be triggered right after the initial encoding of genre-specific acoustic properties [46], which highlights the need for a comprehensive assessment of patient needs and preferences prior to intervention. In addition, the suggestions and professional judgment of music therapists or researchers are vital as they allow better control during the intervention. In the studies analyzed here, most music was chosen by both the patients and the researchers. Music was chosen by only the researchers in 4 studies $[4,11,25,37]$ and by only the patients in 3 studies $[10,13,40]$. Combining the music preference of patients and the professional judgment of music therapists or researchers may be the best choice for the therapeutic relationship and for effective intervention. Future studies may attempt to improve therapeutic strategies by helping patients enjoy the therapeutic process and explore its potential and their confidence in it.

Music intervention was applied with different timing in the included studies. These differences in timing demonstrate that music can be incorporated throughout the course of treatment. Following indications that music intervention has positive effects on chemotherapy-induced anxiety, nausea, and vomiting [24-31], it has become widely used during chemotherapy. Perioperative music can reduce mean arterial pressure, anxiety, and pain among women undergoing mastectomy for breast cancer [13]. A statistical analysis suggested that most patients with late-stage breast cancer choose to undergo mastectomy, radiation, and chemotherapy [47]. After mastectomy, an impaired body image may lead to lack of self-confidence and feelings of inferiority. Music has provided patients with a tool that can be used for emotional adjustment, introspection, and self-exploration to support self-confidence, promote relaxation, and find the courage to confront even more difficult psychological issues [48]. Researchers and music therapists can use different music techniques to promote clinical effects by optimiz- 
ing both the physical and the psychological state of patients during different stages, according to the characteristics of the patients or the equipment available in the therapy room.

Of the included studies, 14 mentioned the long-term effects of music intervention in breast cancer, and all reported positive effects of music on the physical or mental status of breast cancer patients $[3,4,6-9,12,17,26,29,30,37-39]$. This could be strong evidence for the long-term application of music intervention in the clinic.

\section{Quality of the Evidence}

Of the included studies, 9 were considered as high quality, whereas the remaining 21 were of low quality. As the majority of trials were of relatively low quality, the findings of this review need to be interpreted with caution. However, it is known that double blinding of participants is not possible in music interventions, thus none of the included studies received 2 points in the Jadad scale score for this item. In addition, many of the trials in this review included subjective outcomes such as anxiety, pain, and depression. Thus there is a definite danger of bias when participants cannot be blinded to the intervention. Comparative designs and consistent methodology are suggested to reduce the risk of low-quality studies in the future.

\section{Strengths and Limitations}

There are several obvious strengths of this review. First, this study is the first meta-analysis to evaluate the effects of music intervention on patients with breast cancer. Second, this review gives a profound discussion on the application of music and delivers useful advice on how to improve music intervention in future studies, which is unique in the related literature. Third, the review included only RCTs, which limited the observational and selection bias. Forth, all eligible articles were carefully and independently extracted from 9 databases by the 2 first authors to ensure the search results were reproducible. Fifth, a subgroup analysis was conducted to explain the observed heterogeneity.
There are, however, some limitations to this analysis. First, our results retain the potential for unknown publication bias, which we were unable to detect without additional information. Second, we could not conduct a quantitative analysis of pain, nausea, or vomiting because of the limited number of studies and varied scales. Third, heterogeneity is found in our review for methodological diversity. Consequently, we look forward to future efforts and explorations in this field to overcome these deficiencies.

\section{Conclusion}

Our meta-analysis of the effects of music intervention on patients with breast cancer indicates that music intervention is partially effective in improving physiological and psychological symptoms in patients with breast cancer. These results support the use of music in the clinical setting to benefit more patients with breast cancer. Future research should strengthen the analysis of music interventions by standardizing the application of rigorous randomized grouping, using proper allocation concealment, and using professional music therapists in the studies. Music can be a good intervention in breast cancer patients not involving medication. We hope our findings provide valuable information for future music intervention research and promote the implementation of music in the clinic.

\section{Authors' Contributions}

XW: Data search, inclusion and exclusion of studies, data extraction, assessment of methodological quality, main writer. YZ: Data search, inclusion and exclusion of studies, data extraction, assessment of methodological quality, main writer. YF: Selection of projects, guidance for music therapy and systematic review, article revision. X-ST: Data search, meta-analysis. XL: Guidance for systematic review.

\section{Disclosure Statement}

This study was not supported by any funding body. The authors declare that there is no conflict of interest.

\section{References}

1 Torre LA, Bray F, Siegel RL, Ferlay J, Lortettieulent J, Jemal A: Global cancer statistics, 2012. CA Cancer J Clin 2015; 65: 87-108.

2 Benson JR, Jatoi I: The global breast cancer burden. Future Oncol 2012; 8: 697-702.

3 Zhou LJ: Study of effects of personalized music intervention on depression and anxiety among breast cancer patients during chemotherapy period (in Chinese). Master's Thesis, Nursing Department, Central South University, 2009.

4 Yang J, Zhang Q, Gu J, Li L: Effect of Chinese medicine five-tone therapy intervention on the depression state of the chemotherapy patients with breast cancer (in Chinese). Chin J Mod Nurs 2013; 48: 4461-4463.
5 Sun W, Zhao L, Liu M, Xu L: Effects of personalized music intervention on reducing adverse psychological reaction in patients with breast cancer during chemotherapy (in Chinese). Anhui Med Pharm J 2013; 17: 1441-1443.

6 Ren X, Zhou L, Liu L, Guo Q: Effects of personalized music intervention depression in patients of breast cancer during chemotherapy period (in Chinese). Chin J Mod Med 2010; 20: 771-774.

7 Huang Y: Effect of music intervention on the depression mental of post-operative chemotherapy interval patients with breast cancer (in Chinese). Chin J Mod Nurs 2012; 18: 552-554.

8 Fu Y, Dai D, Zhou X, Liang Q: Investigation on the application of music therapy to improve the anxiety and depression of advanced breast cancer chemotherapy with the FEC (in Chinese). Chin J Misdiagnostics 2009; 9: 8312-8313.
Li X, Zhou K, Yan H, Wang D, Zhang Y, Dang S: Effects of music therapy on depression and duration of hospital stay of breast cancer patients after radical mastectomy. Chin Med J 2011; 124: 2321-2327.

10 Zheng Z, Chen P, Wang C: Effects of point massage in combination with music therapy on post-operative pain in patients with breast cancer (in Chinese). Chin Pract Med 2015; 10: 235-236.

$11 \mathrm{Gu} \mathrm{H}$ : Effect of yoga music on anxiety and pain of patient with breast cancer after PICC operation (in Chinese) Today Nurs 2015; 71-72.

12 Li X, Zhou K, Yan H, Wang D, Zhang Y, Dang S: Effects of music therapy on pain among female breast cancer patients after radical mastectomy: results from a randomized controlled trial. Breast Cancer Res Treat 2011; 128: 411-419. 
13 Binns-Turner PG, Wilson LL, Pryor ER, Boyd GL, Prickett $\mathrm{CA}$ : Perioperative music and its effects on anxiety, hemodynamics, and pain in women undergoing mastectomy. AANA J 2011; 79:S21.

14 Hanser SB, Bauer-Wu S, Kubicek L, Healey M, Manola J, Hernandez M, Bunnell C: Effects of a music therapy intervention on quality of life and distress in women with metastatic breast cancer. J Soc Integr Oncol 2006; 4: 116-124.

15 Li Z: Correlations between social psychological factors and life quality of breast cancer patients. Mod Clin Nurs 2009; 12.

16 Bokhari F, Sawatzky JA: Chronic neuropathic pain in women after breast cancer treatment. Pain Manag Nurs 2009; 10: 197-205.

17 Li X, Zhou K, Yan H, Wang D, Zhang Y: Effects of music therapy on anxiety of patients with breast cancer after radical mastectomy: a randomized clinical trial (in Chinese). J Adv Nurs 2012; 68: 1145-1155.

18 Sharpley CF, Bitsika V, Christie DRH: Helping prostate cancer patients understand the causes of anxiety and depression: comparing cancer-caused vs. patient response events. J Mens Health 2009; 6: 345-353.

19 Wu Y, Zhong L, Ye S: Effects of five-tone music therapy on reducing pre-operative anxiety in patients with breast cancer (in Chinese). Fujian Med J 2014; 36: 148-149.

20 Katz J, Poleshuck EL, Andrus CH, Hogan LA, Jung BF, Kulick DI, Dworkin RH: Risk factors for acute pain and its persistence following breast cancer surgery. Pain 2005; 119 : $16-25$.

21 Vega VP: Music therapy and medicine: theoretical and clinical applications. J Music Ther 2004; 41: 186-188.

22 Stanczyk MM: Music therapy in supportive cancer care. Rep Pract Oncol Radiother 2011; 16: 170-172.

23 Liang Y, Jiang X: Effect of music therapy on patients with breast cancer during perioperative period (in Chinese). Chin Community Doct 2010; 12: 46-47.

24 Zhang J, Li Y, Lin N: Effects of music therapy on gastrointestinal reaction in patients with breast cancer (in Chinese). J Med Theory Pract 2016; 29: 689-691.

25 Yao X, Chen M: Effects of angle mode music on nausea and vomiting in patients with breast cancer during chemotherapy period (in Chinese). Nei Mongol J Tradit Chin Med 2015; 6 .
26 Xie J: Effects of music therapy on patients with advanced breast cancer during chemotherapy (in Chinese). Today Nurs 2006; 76-77.

27 Wang Y: Application of music therapy in patients with breast cancer during chemotherapy (in Chinese). J Community Med 2009; 7: 26-27.

28 Wang H, Guo Y: Effects of post-operative music therapy on reducing vomiting in patients with breast cancer during chemotherapy (in Chinese). J Changzhi Med Coll 2011; 25: 392-393.

29 Pan Q, Ma L, Shi L: Effect of individualized music therapy on gastrointestinal reaction in patients with breast cancer (in Chinese). Hainan Med J 2015; 26: 1553-1554.

30 Ma X: Clinical application of playing music in patients with breast cancer resection (in Chinese). Liuzhou Med 2013; 26: 17-19.

31 Jin Y, Hu W, Gu H, Le Y: Effects of music assisted therap on relieving chemotherapy-induced adverse effects of patients with breast cancer operation (in Chinese). Nurs J Chin PLA 2008; 25: 1-3.

$32 \mathrm{Wu} \mathrm{Y}$ : Psychological intervention and influence of background music in modified radical mastectomy for breast cancer (in Chinese). Heilongjiang Med J 2009; 33: 709-710.

33 Xu L, Wang Y: Application of background music in modified radical mastectomy for breast cancer (in Chinese). Chin Mod Doct 2010; 48: 75-76.

34 Lv H, Chen J, Chen J, Peng S, Yang X: Effect of personalized music intervention on the preoperative anxiety in patients undergoing breast cancer surgery (in Chinese). J Nurs Sci 2008; 23: 4-6.

35 Boehm K, Cramer H, Staroszynski T, Ostermann T: Arts therapies for anxiety, depression, and quality of life in breast cancer patients: a systematic review and meta-analysis. Evid Based Complement Alternat Med 2014; 2014: 103297.

36 Bradt J, Dileo C, Grocke D, Magill L: Music interventions for improving psychological and physical outcomes in cancer patients. Cochrane Database Syst Rev 2011; 66: 13051305.
$37 \mathrm{Lu}$ Z, Hu Y: The effect of music relaxation therapy on the adverse reactions induced by chemotherapy in patient with breast cancer (in Chinese). Chin J Nurs 2010; 45: 405408.

38 Chen L, Xie Z, Feng Z, Huang G, Yin Z, Yu Z: Effect of cognitive behavioral intervention therapy on immunological function of patients with breast cancer (in Chinese) Chin J Clin Rehabil 2004; 8: 6310-6311.

39 Chen L, Xie Z, Huang G, Yin Z, Yu Z: The effect of cognitive behavioral therapy on quality of life in patients with breast cancer (in Chinese). Chin J Clin Rehabil 2002; 6 : 2686-2687.

40 Bulfone T, Quattrin R, Zanotti R, Regattin L, Brusaferro S: Effectiveness of music therapy for anxiety reduction in women with breast cancer in chemotherapy treatment. Holist Nurs Pract 2009; 23: 238-242.

41 Jadad AR, Moore RA, Carroll D, Jenkinson C, Reynolds DJ, Gavaghan DJ, McQuay HJ: Assessing the quality of reports of randomized clinical trials: is blinding necessary? Control Clin Trials 1996; 17: 1-12.

42 Review Manager (RevMan) Copenhagen, The Nordic Cochrane Centre, the Cochrane Collaboration, 2008.

$43 \mathrm{Yu} \mathrm{J}$, Xie Q: Music therapy and its application in psychological rehabilitation (in Chinese). Chin J Rehabil Med 2006; 21: 461-463.

44 Altenmüller E, Schlaug G: Neurologic music therapy: the beneficial effects of music making on neurorehabilitation. Acoust Sci Technol 2013; 34: 5-12.

45 Wigram T, Saperston B, West R (eds): Art \& Science of Music Therapy: A Handbook. Chur, Harwood Academic Publishers, 2013.

46 Gjerdingen RO, Perrott D: Scanning the dial: the rapid recognition of music genres. J New Music Res 2008; 37: $93-$ 100.

47 Siegel R, Desantis C, Virgo K, Stein K, Mariotto A, Smith T, Cooper D, Gansler T, Lerro C, Fedewa S: Cancer treatment and survivorship statistics, 2012. CA Cancer J Clin 2012; 62: 220.

48 Bonde LO: Imagery, metaphor and perceived outcomes in six cancer survivors' BMGIM therapy; in Meadows A (ed) Qualitative Inquiries in Music Therapy: A Monograph Series, vol 3. New Braunfels, TX, Barcelona Publishers, 2007. 